\title{
LA ELABORACIÓN DE UN CORPUS DEL PROFESORADO DE ESPAÑOL (COPELE): ¿UTOPÍA O REALIDAD? ${ }^{1}$
}

\author{
THE ELABORATION OF A CORPUS OF SPANISH TEACHERS \\ (COPELE): UTOPIA OR REALITY?
}

\section{MARI MAR BOILLOS PEREIRA}

Universidad del País Vasco-Euskal Herriko Unibertsitatea.

mariadelmar.boillos@ehu.eus

\section{ALBERTO RODRÍGUEZ-LIFANTE}

Universidad de Alicante-Universitat d'Alacant.

alberto.rodriguez@ua.es

\section{RESUMEN}

La lingüística de corpus constituye una rama de la lingüística con un gran potencial para la práctica del docente de lenguas extranjeras. Hasta el momento, son numerosos los corpus en los que se recogen muestras de lengua de estudiantes de lenguas extranjeras con el fin de llevar a cabo intervenciones didácticas orientadas a la mejora del proceso de enseñanzaaprendizaje. Sin embargo, el foco de este estudio siempre se ha localizado en el estudiante y no así en el docente, quien requiere, por su parte, información relativa a las creencias, las percepciones y las actitudes de sus pares. Este trabajo se plantea como objetivo el analizar la posibilidad de crear un corpus del profesorado de ELE. Para ello se ha diseñado y administrado un cuestionario para docentes de perfiles y contextos diversos $(n=130)$ para averiguar el papel que ocupan los corpus en su ejercicio laboral, la percepción que poseen de su propia profesión, así como los beneficios que tendría la existencia de un corpus como CopELE. Los resultados ponen de manifiesto un gran acuerdo de todos los profesionales participantes en relación con los múltiples beneficios que este podría reportar, entre otros, al logro de una mejora del reconocimiento de su práctica docente.

Palabras clave: Lingüística de corpus; español/lengua extranjera; profesorado de lenguas; creencias; competencias docentes.

${ }^{1}$ Parte de este trabajo se ha llevado a cabo gracias al proyecto Competencia existencial del profesorado de español como segunda lengua y lenguas extranjeras (ACIE14-11) en el marco del grupo de investigación de Adquisición y Enseñanza de Segundas Lenguas y Lenguas Extranjeras de la Universidad de Alicante (ACQUA). 


\begin{abstract}
Corpus Linguistics constitutes a branch of linguistics that affects positively the teaching practice of the language teacher. Many corpora, where samples of foreign language students are registered, have been published to carry out pedagogical interventions to improve the learning and teaching process. However, most of these studies are focused on students rather than teachers, who require, on this behalf, information about their peer's beliefs, perceptions, and attitudes. The present study analyzes the possibilities of elaborating a corpus of Spanish teachers. A questionnaire for teachers with different profiles and settings has been designed and administrated $(n=130)$ to find the role of corpus in their teaching practice, the perception of their profession, as well as the benefits that the existence of this corpus (CopELE) would/could have. Results show unanimity among the participants in relation to the benefits this could have, among others, for the acknowledgement of their teaching practice.
\end{abstract}

Keywords: Corpus linguistics; Spanish/foreign language; language teaching; beliefs; teaching skills.

Recibido: 17.11.2017. Aceptado: 04.11.2018.

\title{
1. INTRODUCCIÓN
}

T a Lingüística de Corpus (LC), como uno de los ámbitos relevantes en el área de la enseñanza de segundas lenguas, se ha desarrollado fundamentalmente en tres direcciones: en primer lugar, el empleo de corpus textuales (CT) como fuente de datos cuantitativos para la creación de materiales de enseñanza de lenguas extranjeras $^{2}$ (en adelante, LE); en segundo lugar, el uso de CT en la enseñanza y el aprendizaje de lenguas; $y$, en tercer lugar, la elaboración de los corpus de aprendices (Cruz Piñol, 2012; Rojo, 2015, Alonso-Ramos, 2016). En esta línea, como ha sucedido en los estudios de otras lenguas (como el inglés, con International Corpus of Learner English, Longman Learner Corpus o Cambridge Corpus Project), ha aumentado el interés por elaborar corpus con la finalidad de mejorar la adquisición de los aprendices de español/LE (en adelante, ELE). Así lo atestigua uno de los más recientes sobre aprendientes de español, el Corpus de Aprendices de Español (CAES), elaborado por el Instituto Cervantes (2014).

Ahora bien, aunque todos ellos han abierto grandes posibilidades para el profesorado de lenguas en relación con su alumnado, gran parte de las investigaciones sobre LC han tenido como objeto último el aprendiz, siendo el docente de ELE

\footnotetext{
${ }^{2}$ En este trabajo se ha optado por el empleo del término "lengua extranjera" para hacer referencia a las lenguas no maternas, independientemente de que el proceso de aprendizaje se produzca o no en un contexto de inmersión.
} 
su usuario en términos interpretativos. Por este motivo, ante el papel que la LC ha supuesto para los docentes de lenguas, surge la necesidad de investigar las posibilidades de elaborar un corpus del profesorado de ELE donde tengan cabida aspectos tan diversos como las percepciones, experiencias y actitudes acerca del proceso de enseñanza de español, cuya observación por estos profesionales es difícil, y que son prueba de las competencias que debe desarrollar el profesorado en su ejercicio profesional (Instituto Cervantes, 2012). Esta propuesta, bautizada con el nombre de CopELE (corpus del profesorado de español como lengua extranjera), se desarrolla en el presente artículo a través de una investigación descriptivo-correlacional, que pone de manifiesto su necesidad, y de su fundamentación metodológica.

A partir de estos planteamientos, el objetivo que aquí se persigue es triple: en primer lugar, averiguar el papel que poseen los corpus en el aula de idioma según el profesorado de español; en segundo lugar, explorar cuáles son las concepciones que este profesorado posee sobre su profesión y qué medidas considera que deben adoptarse para mejorar su práctica docente en el aula; y, en tercer lugar, conocer las percepciones que estos docentes presentan sobre la elaboración de un corpus acerca de sus propias creencias.

El CopELE se nutrirá con narrativas escritas y orales elaboradas por docentes de español y podrá enriquecerse a partir de sus contribuciones, procedentes de múltiples contextos geográficos y educativos, y de etapas diferentes de su desarrollo profesional.

En la línea de los objetivos anteriores, este trabajo aborda, en primer lugar, la evolución de la LC en el ámbito de segundas lenguas y su especial desarrollo en ELE. Esta revisión bibliográfica a su vez permite contextualizar la viabilidad del proyecto al que aludimos en el contexto de enseñanza de español. En segundo lugar, se detalla el diseño de la investigación atendiendo a los instrumentos de recogida de datos, así como a los participantes del estudio. En el apartado dedicado a la discusión de resultados se responde a las preguntas de investigación y se analizan los datos obtenidos a la luz de las aportaciones teóricas existentes. Por último, en las conclusiones se presentan algunas de las futuras líneas de actuación, así como las implicaciones y las aplicaciones propedéuticas del proyecto.

\section{MARCO TEÓRICO}

La LC se ha definido como "una rama de la lingüística que basa sus investigaciones en datos obtenidos a partir de corpus, esto es, muestras reales de uso de la lengua" (Martín Peris, 2008, p. 335). Así pues, un corpus puede considerarse "a set of natural texts (or pieces of texts), stored in electronic form, assumed to be jointly representative of a linguistic variety in some of its components, or in all of them, and 
grouped together so that they can be scientifically studied ${ }^{3 "}$ (Rojo, 2015, p. 37).

La utilidad de los corpus como parte de los estudios de segundas lenguas es indiscutible y constituye una reseñable fuente de recursos para identificar los errores de la interlengua de los estudiantes y su codificación atendiendo al tipo de alumnado (lengua materna nivel de dominio de la LE, origen, años de estudio de la LE, experiencias en países hispanohablantes, etc.) (Rojo, 2015). Una ventaja de su uso, como apunta Tolchinsky (2014, p. 15), es el hecho de que, frente a lo que sucedía en el pasado, "actualmente se recopilan con entornos web donde se producen situaciones de comunicación muy abiertas y espontáneas en las cuales se alteran las convenciones normativas de la lengua escrita".

Todo este desarrollo de la didáctica de lenguas extranjeras a partir de los corpus es un planteamiento que cuenta con un largo recorrido, con encuentros internacionales como el TALC (Teaching and Language Corpora) y con la creación de diversos corpus de aprendices de otras lenguas extranjeras (como el inglés, el francés y el español). En el contexto hispanófono no se ha recibido de manera tan positiva como lo ha hecho en otros como el anglosajón, donde ha contado con mayor difusión y el número de contribuciones relacionadas con la formación continua del profesorado es significativo (Tolchinsky, 2014). Ahora bien, ¿̇a qué se debe esta diferencia entre ambos? Según algunos autores, las razones que han contribuido a esta desafección en el ámbito del ELE han venido motivadas por "la relación entre corpus e informatización" (Tolchinsky, 2014, p. 11), la falta de familiarización del profesorado, así como su ausencia en las etapas de formación donde se incluyan como parte fundamental del programa (Pitkowski \& Vásquez, 2009).

A este hecho, cabe añadir los retos a los que los corpus de aprendices deben enfrentarse en un mundo cada vez más global. Meunier (2011) señala la necesidad de recabar corpus en los que se tengan en cuenta más lenguas, más modos comunicativos, más tipos y géneros, más datos de carácter longitudinal y más variables. En relación con este último aspecto, también manifiesta que "more language learning variables should be collected and encoded at the time of corpus collection (proficiency, language aptitude, motivation, more precise description of the task, of temporal, social or situational settings, etc.) ${ }^{4 \prime}$ (Meunier, 2011, p. 467). Resulta significativo, en este sentido, que se aluda al interés por etiquetar otras variables de aprendizaje que pueden estar presentes en los datos que se recaban, pero que pasan desapercibidas para quien los codifica, porque no

\footnotetext{
3 "Un conjunto de textos naturales (o partes de textos), organizado de manera electrónica, que asume ser lo más representativo posible en su variedad lingüística en algunos de sus componentes o en todos ellos, y está agrupado de modo que puede ser científicamente estudiado". Todas las traducciones presentes en el estudio son de elaboración propia.

4 "más variables del aprendizaje de lenguas deben ser recabadas y codificadas en el momento de recoger el corpus (competencia, aptitud lingüística, motivación, una mejor descripción precisa de la tarea, de los contextos temporales, sociales o situacionales, etc.)".
} 
están entre los intereses de la persona que lo lleva a cabo.

Ahora bien, hemos venido planteando a lo largo de estas líneas la importancia de los corpus y su desarrollo para fines pedagógicos. En esta evolución se ha constatado que estos se han centrado en el alumnado como destinatario del proceso de enseñanza. Sin embargo, frente a sus usos indirectos están relacionados con la elaboración de programas y materiales para la enseñanza de lenguas, estos también pueden ofrecer datos muy valiosos sobre cómo enseñar. McEnery y Xiao (2011, p. 14) apuntan a los usos directos de los corpus siguiendo a Leech (1997), a saber, "'teaching about', 'teaching to exploit', and 'exploit to teach"'s. De esta distinción, nos detenemos en el último de ellos, que interpretamos con algunas matizaciones: la explotación de corpus para la enseñanza se podría extender a situaciones en las que los docentes de lenguas se benefician de los contenidos que se recogen en este.

Las funciones didácticas y formativas se hallan estrechamente conectadas en la propuesta de corpus que aquí se plantea y que recibiría, como se ha mencionado, el nombre de CopELE. Esta combinación de elementos se halla en la reciente propuesta de creación de la primera comunidad de práctica del IC, TutorIC, que define como: "una nueva modalidad formativa y de aprendizaje que sirve como espacio de interacción y mediación entre profesionales que comparten intereses y objetivos y donde se reflexiona sobre la práctica profesional y se construye conocimiento de forma conjunta" (Instituto Cervantes, 2017). Se trata, pues, de un espacio dirigido a profesionales en activo que deseen desarrollar su competencia como tutores de prácticas de docentes en formación, colaborando e investigando con compañeros de diferentes contextos profesionales. Una necesidad que los profesionales de español demandan desde hace tiempo de la mano de un discurso más internacional de la enseñanza de español con beneficios para el avance de la investigación y la mejora de la formación, con implicaciones y aplicaciones prácticas en la enseñanza de español (Muñoz-Basols, Muñoz-Calvo y Suárez-García, 2014).

Por ello, traemos a colación en estas líneas a la figura del docente de lenguas y su faceta de investigador en acción. El desarrollo de su práctica reflexiva precisa tanto del conocimiento de los corpus y sus aplicaciones didácticas, como del aumento y contraste de las percepciones que posee sobre múltiples aspectos en los procesos de enseñanza y aprendizaje. Así pues, existe una necesidad cada vez mayor por parte del profesorado de lenguas (en formación o con mayor experiencia; nativo o no nativo; interesado en la investigación o preocupado por mejorar su enseñanza) de acceder a todos aquellos interrogantes que emergen en su práctica docente y que, por su naturaleza, son de difícil consulta o que no suelen ser compartidos por su carácter personal. Asimismo, conviene destacar la situación del profesorado de español en el mundo actual, donde la variación de su centro o institución, su país, grupo de estudiantes meta o cultura poseen gran relevancia.

5 “'enseñanza sobre', 'enseñanza para explotar', y 'explotar para enseñar”. 
Todas estas cuestiones conducen a plantearse si las creencias, las percepciones y las actitudes que nacen en estos procesos pueden ser de utilidad para una mejora de la comprensión de todos los aspectos complejos y dinámicos que forman parte de la enseñanza y el aprendizaje de idiomas (Ellis, 2008; De Bot y Larsen-Freeman, 2011; Larsen-Freeman, 2015). En palabras de Stubss (2001, apud Tolchinsky, 2014, p. 15), "al igual que los geólogos, que exploran las rocas porque están interesados en los procesos geológicos a los que no tienen acceso directo, los investigadores en adquisición y los profesores de lengua pueden explorar los datos de los aprendices para inferir lo inaccesible: los procesos mentales de adquisición de las lenguas".

Esos procesos mentales afectan también al profesorado y a sus percepciones. Ya en la década de los 70, Argyris \& Schön (1974, p. 30) afirmaban que "casi siempre existe una discrepancia entre lo que los profesionales dicen que creen (sus teorías 'asumidas') y sus formas de actuar (sus 'teorías en acción')". En este camino exploratorio, el profesorado debería desarrollar una faceta reflexiva para ser capaz de adaptarse y dar respuesta a las demandas que tanto dentro como fuera del aula de idiomas tendrán lugar.

También, relacionado con este planteamiento, surge todo un sistema de creencias que el profesorado va desarrollando a lo largo de su vida. Williams y Burden (1999, p. 65) subrayan que el docente "se deja influir enormemente por las creencias, que a su vez están íntimamente unidas a sus valores, a su visión del mundo y al concepto que tienen de su posición en el mismo". Este hecho pone de manifiesto tanto la relevancia que poseen en dicho proceso, como la necesidad de llevar a cabo un análisis más profundo de todas estas percepciones, a pesar de la dificultad que entraña su exploración y definición, ya que todas ellas están relacionadas con todo lo que tiene lugar en el aula.

En este caso, todo el conjunto de valores, percepciones y creencias no solo afectan al alumnado, sino también al proceso de aprendizaje y al profesorado. Conviene incidir en este último aspecto, ya que nos ayuda a plantearnos de qué modo podría profundizarse en estos elementos que se entrelazan con la dimensión social, afectiva y emocional de su profesión. Si nos detenemos a pensar en las etapas en las que gran parte de estos aspectos se desarrollan, podemos apuntar a aquellas en las que hemos experimentado el aprendizaje de una lengua. Ahora bien, no siempre se llevan a cabo reflexiones acerca de esas experiencias como aprendices de lenguas, de modo que difícilmente nos planteamos su importancia cuando dejamos de aprender para enseñar. Este es el punto de partida en el que se basa el estudio de Breyer (2009) donde explora, precisamente, las reflexiones de estudiantes que se convertirán en futuros profesores de lengua (en este caso de inglés) acerca del uso de los corpus como herramienta pedagógica en el aula y de formación del profesorado. Los resultados indican que los participantes no solo adquieren una mayor conciencia lingüística, sino también un vínculo más fuerte entre la lengua que enseñan y el modo de hacerlo (Breyer, 2009). 
En palabras de Williams y Burden (1999, p. 65), "si el profesor como educador es el que evalúa constantemente, a la luz de los nuevos conocimientos, sus creencias sobre el lenguaje o sobre la forma de enseñarlo o sobre la educación en general, entonces es fundamental que los profesores entiendan y articulen primero sus propias perspectivas teóricas".

A la vista de estas afirmaciones, pueden comprobarse todos estos aspectos a los que alude la "competencia existencial", tal y como se recoge en el Marco común europeo de referencia para las lenguas: aprendizaje, enseñanza y evaluación (en adelante, Marco) (Ministerio de Educación, Cultura y Deporte, 2002), en otros documentos como el Marco de Referencia para los Enfoques Plurales de las Lenguas y las Culturas (MAREP) (CARAP en francés) (Candelier et al., 2008), el Plan Curricular del Instituto Cervantes (PCIC) (Instituto Cervantes, 2007), así como diversos informes sobre el profesorado (Kelly, Grenfell, Allan, Kriza y McEvoy, 2004; Newby, Allan, Fenner, Jones, Komorowska y Soghikyan, 2007; Instituto Cervantes, 2011; Instituto Cervantes, 2012), donde se señalan algunas líneas de actuación relevantes para su desarrollo profesional. Sin embargo, a la complejidad que entraña el explorarlas y analizarlas se añade muchas veces la reticencia del profesorado a hacer públicas o compartir este tipo de reflexiones personales.

Este hecho nos conduce a plantear la necesidad de elaborar un corpus donde se recojan diarios de aprendizaje, narraciones e historias sobre los procesos de enseñanza. Es decir, un espacio de reflexión e interacción entre diversos contextos, niveles, metodologías, entornos de instrucción y percepciones. De este modo, el docente se presenta como verdadero protagonista para dar voz a esas creencias que están presentes en las aulas de español y cuya presentación como parte de un corpus de narrativas puede aportar un valor añadido a la enseñanza. Asimismo, para aquellas personas que investiguen procesos en segundas lenguas, puede resultar de gran interés como fuente de datos sobre los factores existenciales del profesorado (creencias, motivaciones, actitudes, valores y otros factores) (Martín Peris, 2008; Rodríguez-Lifante, 2016) ${ }^{6}$.

Hasta el momento, todos estos procesos internos, inherentes a la enseñanza de una LE, son de difícil acceso para los profesionales de este sector, ya que gran parte de esta información está recogida en trabajos de investigación, como trabajos de fin de máster o tesis doctorales, pero su consulta a menudo queda condicionada a su publicación en papel o en red. Una posible solución es la que señala Rojo (2015, p. 28) al referirse "las enormes posibilidades que brinda la existencia de la red, de la cual deriva la línea conocida como Web as corpus". A pesar de todo ello, y sin menoscabar el papel que la red posee en la divulgación de esta información,

\footnotetext{
${ }^{6}$ Estos factores forman parte de la competencia existencial (saber ser), definida como "la suma de las características individuales y rasgos de personalidad que se relacionan con la imagen que uno tiene de sí mismo y de los demás, así como con la voluntad de entablar una interacción social con otras personas" (Martín Peris, 2008, p. 105).
} 
se observa la necesidad de dar luz a una base de datos abierta y anónima donde los profesionales de la enseñanza de lenguas puedan contribuir y compartir todos estos procesos que se desarrollan en el aula de español y que, hasta ahora, no han recibido la necesaria atención desde la LC.

Para ello, la técnica de las narrativas escritas y orales, empleadas en numerosas investigaciones cualitativas de segundas lenguas (Oxford y Green, 1996; Kalaja, Menezes, y Barcelos, 2008; Dörnyei, Henry y Muir, 2016), responden a los objetivos perseguidos en este proyecto. En este sentido, este instrumento de recogida permite registrar numerosos factores, que serían analizados y categorizados posteriormente, en función del interés de cada persona. La inexistencia de trabajos que hayan explorado esta posibilidad en relación con el español como LE contrasta con la del inglés. Es el caso del proyecto AMFALE (Aprendiendo con hablantes y aprendices de lenguas extranjeras) (2002-2003), coordinado por la profesora Menezes en Brasil, que consiste en un corpus de 600 narrativas sobre experiencias de aprendizaje de estudiantes y profesorado de inglés en formación de diferentes países (Menezes, 2017). El equipo encargado del análisis de las narrativas se centró en diversos aspectos (creencias de los aprendices, cuestiones culturales, errores, experiencias, identidades, competencias, influencia de la instrucción formal, procesos de adquisición de la lengua inglesa en la memoria del alumnado de enseñanza media, autonomía de aprendizaje de una LE o la adquisición del portugués como LE, entre otros aspectos). Asimismo, un corpus oral sobre el discurso del profesorado coreano de inglés como lengua extranjera en el aula de idiomas se está llevando a cabo también en la actualidad (Centre for English Corpus Linguistics, 2017). Ambos ejemplos ponen de manifiesto el interés cada vez mayor por la figura del docente en la elaboración de corpus.

\section{METODOLOGÍA Y DISEÑO DE LA INVESTIGACIÓN}

En la metodología empleada para llevar a cabo el presente estudio, se alude a las preguntas de investigación que hemos perseguido responder, así como a las hipótesis de partida. A continuación se explica el diseño de la herramienta empleada para la obtención de datos, el procedimiento para la recogida de los mismos y el perfil de los participantes de este estudio.

\subsection{Preguntas de investigación e hipótesis de partida}

Una revisión de los conceptos teóricos en los que se sustenta el presente estudio y de las investigaciones previas realizadas en torno a la viabilidad de la elaboración de un corpus de creencias, percepciones y actitudes del profesorado, han conduci- 
do a plantear las siguientes tres preguntas de investigación:

¿Cuál es el papel de los corpus por el profesorado en el aula de español?

Esta pregunta busca conocer qué presencia poseen los corpus en el ejercicio docente en el aula de ELE y qué manejo acreditan los profesionales acerca de estas herramientas.

¿Qué consideración posee el docente sobre su profesión y qué medidas cree que debe adoptar para la mejora de su práctica docente en el aula?

Mediante esta pregunta de investigación se persigue averiguar cuáles son las concepciones que el propio docente tiene sobre su práctica laboral y qué recursos considera que existen para la mejora de su ejercicio y, en última instancia, para su profesionalización.

¿Qué beneficios posee la elaboración de un corpus del y para el profesorado de españoll LE?

Este tercer interrogante busca conocer las percepciones sobre los beneficios que reporta la creación del CopELE en la práctica docente, así como en el desarrollo de las diferentes competencias que son consideradas como básicas para el docente de ELE.

Con respecto a estas preguntas de investigación, las hipótesis de las que se parte son las siguientes:

H1. Los docentes conocen el uso de los corpus en relación al alumnado, pero no dominan su uso.

H2. Los docentes abogan por la profesionalización de su ejercicio profesional y valoran la creación de recursos que permitan alcanzar este fin.

H3. El profesorado considera que la aplicación de un corpus a su práctica docente y formativa incidiría de manera positiva en el desarrollo de las diferentes dimensiones que configuran la competencia del docente de lenguas.

\subsection{Instrumento para la recogida de datos}

Las preguntas de investigación previamente expuestas dieron lugar a la elaboración de un breve cuestionario organizado en dos apartados fundamentales. El pri- 
mero tenía como objetivo recoger datos en torno al perfil general del participante: sexo, conocimientos lingüísticos, trayectoria docente (años, entorno y perfil del estudiante) y su experiencia en el ámbito de la investigación en ELE. El segundo de ellos estaba compuesto por los ítems relativos a las preguntas de investigación. Los informantes tuvieron que mostrar su grado de acuerdo o desacuerdo con respecto a estos a través de una escala Likert de 1 a 5 , siendo 1 total desacuerdo y 5 total acuerdo.

Los ítems, por razones temáticas, fueron organizados en tres bloques diferentes (Ver Anexo). El primero de estos estaba constituido por 10 preguntas acerca de las necesidades de crear un corpus de las creencias del profesorado. El segundo de los bloques, con 9 ítems, planteaba cuestiones relativas a la utilidad de un corpus de profesorado de ELE. El tercero estaba formado por 32 ítems correspondientes a las subcompetencias del profesorado establecidas por el Instituto Cervantes (2012). En este caso, se perseguía que los informantes indicasen en qué medida consideraban que la creación de un corpus de las percepciones, las actitudes y las actuaciones del profesorado de ELE podía afectar al desarrollo de dichas subcompetencias. Por último, se incluyó una pregunta abierta en la que los informantes podían aportar su punto de vista acerca del objetivo de este estudio o cualquier otra aportación complementaria que se considerase pertinente.

Una vez establecidos los ítems de este estudio, se generó un breve cuestionario en línea que se suministró a través de diferentes redes sociales y listas de distribución a lo largo del mes de mayo de 2016. El objetivo principal era difundir el cuestionario entre diferentes redes y entornos de trabajo para contar con la participación de profesionales con diversidad de perfiles y contextos de modo que se obtuviera una visión más amplia de la realidad docente. El objetivo de este estudio buscaba obtener unas primeras valoraciones de docentes con perfiles dispares, pero que se desenvuelven en diferentes redes profesionales. No obstante, cabe indicar que se ha verificado la fiabilidad de esta escala a través del cálculo del Alfa de Cronbach, siendo este de un,957.

\subsection{Participantes del estudio}

El tipo de muestreo utilizado en la investigación ha sido probabilístico. El criterio utilizado para la muestra tenía en cuenta a docentes de cualquier contexto geográfico y educativo que impartiera ELE. En la presentación del instrumento se describía brevemente el propósito de la investigación y los destinatarios del estudio. Asimismo, la primera pregunta del cuestionario permitía discriminar si la persona participante podía formar parte de ese estudio o no (Ver Anexo). 
El perfil de los participantes de este estudio $(n=130)$ pone de manifiesto algunos aspectos relevantes. En primer lugar, cabe reseñar la notablemente superior participación de las mujeres con respecto a la de los hombres (78,5\%). Una gran mayoría de informantes $(65,4 \%)$ señala el español como, al menos, una de sus lenguas maternas.

En relación a la trayectoria profesional, se observa que un 71,5\% de los participantes poseen una experiencia superior a cinco años en el ámbito de la enseñanza de ELE. Asimismo, resalta el número de participantes cuya actividad profesional en la enseñanza de español se ha desarrollado en diferentes ámbitos (55,4\%), entre los que se hallan las ONG, las enseñanzas primarias o la universitaria. Es precisamente en este último, en el que se encuentra el mayor número de docentes con dedicación exclusiva (14,6\%). Así también, aseguran, en más de un 35\% de los casos, que han ejercido la docencia en diversas áreas geográficas, siendo Europa aquella donde más profesionales la han ejercido de manera exclusiva (40,8\%).

\section{DISCUSIÓN DE LOS RESULTADOS}

Para el cálculo de los resultados de este estudio se tomaron en consideración todos los datos estadísticos recogidos a través del cuestionario. Para ello, tras la comprobación de su Alfa de Cronbach, los ítems se agruparon en función de su contenido temático para dar respuesta a las preguntas de investigación planteadas. Así, la distribución de estos enunciados da como resultado la Tabla I.

Tabla I. Relación de preguntas de investigación con los ítems que corresponden a dichas preguntas. Fuente: elaboración propia.

\begin{tabular}{|l|l|l|}
\hline $\begin{array}{l}\text { Pregunta de } \\
\text { investigación }\end{array}$ & Bloque & Ítems \\
\hline PI.1. & $\begin{array}{l}\text { (A) El uso de corpus en el ámbito de la enseñanza } \\
\text { de ELE }\end{array}$ & $1,2,3,4,5,9,10$ \\
\hline PI.2. & $\begin{array}{l}\text { (A) El uso de corpus en el ámbito de la enseñanza } \\
\text { de ELE }\end{array}$ & $6,7,8$ \\
\cline { 2 - 3 } & $\begin{array}{l}\text { (B) La elaboración de un corpus del profesorado } \\
\text { de ELE }\end{array}$ & $1,2,4,5,6,7,9$ \\
\hline PI.3 & $\begin{array}{l}\text { (B) La elaboración de un corpus del profesorado } \\
\text { de ELE }\end{array}$ & 3,8 \\
\cline { 2 - 3 } & $\begin{array}{l}\text { (C) Los beneficios de un corpus del profesorado } \\
\text { de ELE }\end{array}$ & Todas \\
\hline
\end{tabular}


A continuación, en forma de respuesta a las preguntas de investigación planteadas, se presenta el análisis de los resultados.

\section{¿Cuál es el papel de los corpus por el profesorado en el aula de español?}

Los resultados señalan que este profesorado, a pesar de ser consciente de la existencia de diferentes corpus vinculados al ámbito de ELE ( $\underline{\underline{\bar{x}}} 3.2, \sigma 1.260)$, no parecen emplearlos con normalidad ( $\underline{\underline{x}} 2.49, \sigma 1.348)$ y en su mayoría desconoce sus múltiples beneficios en la enseñanza y el aprendizaje en el aula de idiomas ( $\underline{\underline{x}}$ $2.98, \sigma 1.364)$. El análisis de los datos muestra que no existe una relación directa entre la falta de formación y el uso de esta herramienta con fines pedagógicos ( $\mathrm{r}=$ ,524). Por lo tanto, el hecho de que los corpus no estén presentes en el aula no se debe a que los docentes no hayan recibido esta formación específica. Más bien, como apuntan algunos autores (Pitkowski \& Vásquez, 2009), la gran ausencia en su empleo como parte del profesorado está relacionada con la naturaleza informática y la falta de familiarización con su contenido.

En este sentido, Tolchinsky (2014) señala que, en otros contextos de enseñanza donde se insiste en la necesidad de una formación pedagógica en corpus, como Estados Unidos, Australia y Gran Bretaña, desde hace más de una década se están llevando a cabo investigaciones en las que se recogen numerosas experiencias en la formación continua de los docentes de lenguas (Allan, 1999; Conrad, 2000; Seidlhofer, 2000, 2002), así como propuestas para incluir en la formación inicial del profesorado de lenguas (O'Keeffe y Farr, 2003).

Por otro lado, el análisis de los datos ha permitido comprobar que parece existir una correlación parcialmente significativa entre el grado de conocimiento de los participantes acerca del uso y la utilidad de los corpus y su participación en investigaciones vinculadas a la enseñanza de $\operatorname{ELE}(r=, 026)$. En otras palabras, se podría afirmar que los docentes que tienen un interés por investigar en este campo y por indagar en cómo mejorar el proceso de enseñanza-aprendizaje de ELE, muestran también un interés por conocer cuáles son las potencialidades de los corpus y cómo pueden emplearse con fines pedagógicos.

Parece reseñable, además, que los participantes de este estudio muestran un alto grado de acuerdo ( $\underline{\underline{\bar{x}}} 4.20, \sigma .968)$ al considerar que, si existiera un corpus que recogiese las creencias, las percepciones y las prácticas reflexivas del profesorado de ELE, lo utilizarían como fuente de consulta. De hecho, consideran estas herramientas como esenciales en el proceso de enseñanza de una lengua extranjera. En definitiva, se entendería como un espacio colaborativo que ofrece recursos al docente para lograr un alto nivel de dominio en su actividad profesional y, a su vez, "involucra sus conocimientos, creencias, habilidades en diversos campos, destrezas, actitudes y valores" (Mendoza, 2008, p. 318). 
¿Qué consideración posee el docente sobre su profesión y qué medidas cree que debe adoptar para la mejora de su práctica docente en el aula?

Son varios los aspectos que cabría destacar con respecto a cuál es la percepción que tiene el docente sobre su práctica profesional, así como sobre qué considera que debería hacer para mejorar su ejercicio docente. En primer lugar, los resultados coinciden al afirmar que, en la actualidad, existe un bajo reconocimiento social de la profesión ( $\underline{\underline{\underline{x}}} 2.58, \sigma 1.018)$. En este sentido, se apunta a la investigación como herramienta para elevar el estatus del docente y para consolidar su valía para la enseñanza de español; así también para conocer cuáles son las prácticas que llevan a cabo los compañeros y poder enriquecerse de las experiencias realizadas por iguales. Esta idea se apoya en la importancia que los informantes otorgan a la realización de investigaciones en este ámbito ( $\underline{\underline{x}} 4.25, \sigma 1.042)$.

Estas voces de tantos profesionales de la enseñanza de español se unen a muchas otras que señalan la imperante necesidad de generar un diálogo científico y académico de alto nivel en ELE, al mismo tiempo que se sigue desarrollando una mayor investigación interdisciplinar desde el aula de ELE (Tolosa \& Yagüe, 2009). Así también, se anima a caminar hacia una internacionalización de la investigación sobre el aprendizaje y la enseñanza de ELE (Muñoz-Basols, Muñoz-Calvo y Suárez-García, 2014).

Por su parte, en relación con las herramientas más empleadas para la práctica docente, se observa un frecuente uso de los diarios de aula o diarios de reflexiones $(\underline{\underline{x}} 3.83, \sigma 1.195)$ a través de los que el profesorado recoge sus experiencias llevadas a cabo durante la clase, los aspectos que pueden mejorarse o las necesidades diagnosticadas, entre otras. Este resultado va unido al del interés del docente por las variables individuales del alumnado, así como por el de las opiniones de otros compañeros de profesión sobre su práctica en el aula.

Como se ha señalado en la introducción, a pesar de la consideración de la red como corpus (web as corpus) que ofrece un gran potencial tanto para el alumnado, como el profesorado, la consulta de la redes sociales y otros entornos colaborativos que brindan las telecomunicaciones, no es especialmente relevante para conocer aspectos relevantes sobre opiniones, experiencias y actitudes sobre el proceso de aprendizaje en diversos niveles o contextos geográficos ( $\underline{\underline{\underline{x}}} 3.41, \sigma .994)$, sino que en gran medida suele responder fundamentalmente a necesidades concretas relacionadas con la metodología, los materiales y recursos o los procesos administrativos. Por esta razón, no es de extrañar que desde el ámbito de la formación del profesorado de ELE se estén fomentando comunidades de aprendizaje orientadas a los docentes para compartir y reflexionar de manera conjunta (Instituto Cervantes, 2017). 
¿Qué beneficios posee la elaboración de un corpus del y para el profesorado de españoll LE?

Como ya se ha venido apuntando, los docentes participantes en este estudio coinciden en que los corpus del profesorado pueden ser una herramienta de utilidad para la obtención de un mayor estatus profesional, así como para la mejora de su práctica docente. En este sentido, la respuesta a esta pregunta es de gran relevancia para las competencias docentes de los profesionales de español (Instituto Cervantes, 2012), ya que los datos obtenidos ponen de manifiesto que la elaboración del corpus propuesto tendría implicaciones positivas en las competencias del profesorado.

Tal y como se puede observar en la Tabla II, según los participantes, el empleo de un corpus de creencias, prácticas y percepciones del profesorado (CopELE) sería beneficioso de manera general para el desarrollo de las consideradas como competencias clave (Instituto Cervantes, 2012). De todas ellas, destacan sobre todo las relacionadas con el desarrollo de la competencia profesional y de la evaluación, que incluyen subcompetencias como la capacidad para garantizar buenas prácticas en la evaluación, la habilidad para servirse de las herramientas y procedimientos de evaluación adecuados, la destreza para analizar y reflexionar sobre la práctica docente y la participación activa en el desarrollo de la profesión, entre otros aspectos. Se confirman, por tanto, en este punto de la investigación, unos resultados acordes a los ya obtenidos en las preguntas previas.

Tabla II. Resultados de la escala likert con respecto a la valoración de los beneficios del corpus en relación a las competencias del profesorado.

\begin{tabular}{|l|l|l|}
\hline Competencia & Media & $\begin{array}{l}\text { Desviación } \\
\text { estándar }\end{array}$ \\
\hline Desarrollarse profesionalmente como profesor de la institución & 4,17 &, 773 \\
\hline Evaluar el aprendizaje y la actuación del alumno & 4,00 &, 819 \\
\hline Organizar situaciones de aprendizaje & 3,95 &, 803 \\
\hline Facilitar la comunicación intercultural & 3,94 &, 976 \\
\hline Servirse de las TIC para el desempeño de su trabajo & 3,91 &, 997 \\
\hline Implicar a los alumnos en el control de su propio aprendizaje & 3,87 &, 926 \\
\hline $\begin{array}{l}\text { Gestionar sentimientos y emociones en el desempeño de su } \\
\text { trabajo }\end{array}$ & 3,73 & 1,002 \\
\hline Participar activamente en la institución & 3,66 & 1,058 \\
\hline
\end{tabular}


La distinción entre competencias centrales del docente de idiomas y otras que se extienden a otros profesionales de la enseñanza en general (Instituto Cervantes, 2012) es significativa para llamar la atención sobre la importancia de estrechar lazos entre los profesionales de la enseñanza de español independientemente de la institución, entorno de instrucción o contexto geográfico a los que pertenezcan.

Es importante, además, destacar que, tras la realización de una comparación de medias, se ha observado que ni la experiencia en enseñanza de ELE, ni los ámbitos en los que se ejerce la docencia afectan en los resultados obtenidos en esta pregunta de investigación. Este hecho refrenda no solo la importancia de estas competencias en diversos contextos de enseñanza y con distintas trayectorias formativas, sino también el interés que entre esta variedad de participantes despierta el desarrollo de una comunidad abierta y más amplia de intercambio de reflexiones sobre los procesos de enseñanza, con el objetivo de crear un mayor y mejor conocimiento en torno al ámbito de la enseñanza y el aprendizaje de ELE.

\section{CONCLUSIONES}

Los resultados obtenidos en este estudio apuntan a que existe un elevado grado de acuerdo entre los docentes de español de diversos perfiles, al afirmar que la creación de un corpus como CopELE podría repercutir de manera positiva, tanto en la mejora del ejercicio docente, como en el reconocimiento social de esta profesión. Asimismo, se constata, en líneas generales y a pesar de contar con las percepciones de docentes con diversidad de experiencias, formación y contextos, una falta de conocimiento de las diversas potencialidades que un corpus puede reportar a la práctica docente, mientras se coincide en que un corpus que integrase las creencias, las percepciones y las prácticas reflexivas del profesorado facilitaría el diálogo entre profesionales y generaría pasos hacia la internacionalización del discurso científico de este ámbito.

Además, los resultados de esta investigación coinciden con la literatura al poner de manifiesto el uso que el profesorado hace de la web y las redes sociales como recurso para mejorar y contrastar su práctica docente. Este hecho confirma la preocupación que docentes de español, desde diferentes contextos geográficos, tienen por enriquecerse profesionalmente. Por su parte, se sostiene que las implicaciones positivas de este corpus afectarían a las concebidas como competencias básicas del profesorado de español, fundamentalmente a las vinculadas con la evaluación.

En definitiva, la variedad de perfiles, contextos de enseñanza y antecedentes de los participantes y los resultados obtenidos coinciden en poner de manifiesto las potencialidades de la creación de un corpus y alientan la realización de una segunda fase de este proyecto que consistirá en el diseño de una prueba piloto del denominado CopELE a través de un corpus de narrativas. El objetivo, en esta 
segunda fase, será el crear una herramienta válida que permita, de manera rápida y accesible, obtener información acerca de las creencias, las percepciones y las prácticas del profesorado según diversas variables. Por medio de pruebas de validación y pilotaje de los instrumentos, se podrá investigar las herramientas que permiten hacer un mejor registro y consulta de información de gran relevancia para la mejora de la enseñanza impartida por el profesorado de ELE, así como de su formación.

\section{REFERENCIAS}

Allan, Quentin. (1999). Enhancing the language awareness of Hong Kong teachers through corpus data. Journal of Technology and Teacher Education, 7(1), $57-74$.

Alonso-Ramos, Margarita. (2016). Current Trends and Future Perspectives. Amsterdam/Philadelphia, Netherland/United State: John Benjamins.

Argyris, Chris \& Schön, Donald. (1974). Theory in Practice. Increasing Professional Effectiveness. San Francisco, United State: Jossey-bass.

Breyer, Yvonne. (2009). Learning and teaching with corpora: reflections by student teachers. Computer Assisted Language Learning , 22(2), 153-172.

Candelier, Michael. (coord.), Camilleri-Crima, Antoinette, Castellotti, Verónique, De Pietro, Jean-François, Lőrincz, Ildikó, Meißner, Franz-Joseph, Noguerol, Artur \& Schröder-Sura, Anaa. (2008). Marco de referencia para los enfoques plurales de las lenguas y las culturas (MAREP). Graz: ECML.

Centre for English Corpus Linguistics. (2017). Learner Corpora Around the World. Disponible en https://uclouvain.be/en/research-institutes/ilc/cecl/learner-corpora-around-the-world.html

Conrad, Susan. (2000). Will corpus linguistics revolutionize grammar teaching in the 21st century? TESOL Quarterly, 34, 548-560.

Cruz Piñol, Mar. (2012). Lingüistica de corpus y enseñanza del español como 2/L. Madrid, España: Arco Libros.

De Bot, Kees \& Larsen-Freeman, Diane. (2011). Researching second language development from a dynamic systems theory perspective. En M. Vespoor, K. de Bot, \& W. Lowie, A Dynamic Approach to Second Language Development: Methods and Techniques (pp. 5-23). Amsterdam, Netherland: John Benjamins.

Dörnyei, Zoltán, Henry, Alastair \& Muir, Christine. (2016). Motivational Currents in Language Learning: Frameworks for Focused Interventions. New York, United State: Routledge.

Ellis, Nick. (2008). The dynamics of second language emergence: cycles of language use, language change, and language acquisition. The Modern Language Journal, 92(2), 232-249.

Instituto Cervantes. (2007). Plan Curricular del Instituto Cervantes. Niveles de re- 
ferencia para el español. Madrid, España: Biblioteca Nueva.

Instituto Cervantes. (2011). ¿Qué es ser un buen profesor o una buena profesora del Instituto Cervantes? Análisis de las creencias del alumnado, profesorado y personal técnico y directivo de la institución. Madrid, España: Instituto Cervantes.

Instituto Cervantes. (2012). Las competencias clave del profesorado de lenguas segundas y extranjeras. Madrid: Instituto Cervantes.

Instituto Cervantes. (2014). Corpus de aprendices de español (CAES).

Instituto Cervantes. (2017). Comunidad de práctica: Tutor IC. Actividades formativas Cervantes Disponible en https://cfp.cervantes.es/recursos/proyectos/ comunidades_de_practica.htm

Kalaja, Paula, Menezes, Vera \& Barcelos, Ana. (2008). Narratives of Learning and Teaching EFL. London, England: Palgrave MacMillan.

Kelly, Michael, Grenfell, Michael, Allan, Rebecca, Kriza, Christine \& McEvoy, William. (2004). Perfil Europeo para la Formación de Profesores de Idiomas. Un marco de referencia. Southampton, England: Consejo de Europa.

Larsen-Freeman, Diane. (2015). Complexity theory. En B. Van Patten, \& J. Williams, Theories in Second Language Acquisition. New York, United State: Routledge.

Leech, Geoffrey. (1997). Teaching and language corpora: A convergence. En A. Wichmann, S. Fligelstone, T. McEnery, \& G. Knowles, Teaching and Language Corpora (pp. 1-23). London, England: Longman.

Martín Peris, Ernesto. (coord.) (2008). Diccionario de términos clave de ELE. Madrid, España: SGEL.

McEnery, Tony \& Xiao, Richard. (2011). What corpora can offer in language teaching and learning. En E. Hinkel, Handbook of Research in Second Language Teaching and Learning (pp. 364-380). New York, United State: Routledge.

Mendoza, Silvia Yreri. (2008). Perfil de profesores de lenguas basado en competencias. Una reflexión en torno de formación de profesores. En Memorias del IV Foro Nacional de Estudios de Lenguas (pp. 316-322).

Menezes, Vera. (2017). Aquisiçao em memorias de falantes e de aprendizes de linguas estrangeiras. Disponible en www.veramenezes.com/amfale2.htm

Meunier, Fanny. (2011). Corpus linguistics and second/foreign language learning: exploring multiple paths. Revista Brasileira de Lingüistica Aplicada, 11(2), 459477.

Ministerio de Educación, Cultura y Deporte. (2002). Marco común europeo de referencia para las lenguas: aprendizaje, enseñanza, evaluación. Madrid, España: Anaya.

Muñoz-Basols, Javier, Muñoz-Calvo, Micaela \& Suárez-García, Jesús. (2014). Hacia una internacionalización del discurso sobre la enseñanza del español como lengua extranjera. Journal of Spanish Language Teaching, 1(1), 1-14.

Newby, David, Allan, Rebecca, Fenner, Anne-Brit, Jones, Barry, Komorowska, 
Hanna, \& Soghikyan, Kristine. (2007). European Porfolio for Student Teachers of Languages. A reflection tool for language teacher education. Graz: Consejo de Europa.

O'Keeffe, Anne \& Farr, Fiona. (2003). Using language corpora in initial teacher education: pedagogic issues and practical applications. TESOL Quarterly, 37(3), 389-418.

Oxford, Rebecca \& Green, John. (1996). Language learning histories: learners and teachers helping each other understand learning styles and strategies. TESOL Journal , 6(1), 20-23.

Pitkowski, Elena \& Vásquez, Javier. (2009). El uso de corpus lingüísticos como herramienta pedagógica para la enseñanza y aprendizaje de ELE. Tinkuy (11), $31-51$.

Rodríguez-Lifante, Alberto. (2016). La competencia existencial en el profesorado nativo/no nativo de ELE. En O. Cruz Moya (ed.), La formación y competencias del profesorado de ELE. Granada, España: ASELE, 901-910.

Rojo, Guillermo. (2015). Hispanic corpus linguistic. En M. Lacorte, The Routledge Handbook of Hispanic Applied Linguistic (pp. 371-387). New York, United State: Routledge.

Seidlhofer, Barbara. (2000). Operationalizing intertextuality: using learner corpora for learning. En L. Burnard, \& T. Mcenery, Rethinking Language Pedagogy from a Corpus Perspective (pp. 207-224). New York, United State: Peter Lang.

Seidlhofer, Barbara. (2002). Pedagogy and local learner corpora: working with learning driven data. En S. Granger, J. Hung, \& S. Petch-Tyson, Computer Learner Corpora, Second Language Acquisition and Foreign Language Teaching (pp. 213-234). Philadelphia, United State: John Benjamins.

Tolchinsky, Liliana. (2014). El uso de corpus lingüísticos como herramienta pedagógica. Textos de Didáctica de la Lengua y la Literatura (65), 9-17.

Tolosa, Juan \& Yagüe, Agustín. (2009). Entrevista plural: 25 años de ELE. marcoELE (9), 1-21.

Williams, Marion \& Burden, Robert L. (1999). Psicología para profesores de idiomas. Enfoque del constructivismo social. Madrid, España: Edinumen. 


\section{ANEXO \\ Cuestionario sobre el CopELE \\ Corpus del Profesorado de Español/LE - CopELE}

*Obligatorio

\section{¿Quiénes somos?}

Somos dos profesores que trabajamos en centros de enseñanza superior y que estamos especializados en el ámbito del español/lengua extranjera. Nuestras líneas de investigación se centran en la búsqueda de avances que contribuyan a la mejora del proceso de enseñanzaaprendizaje de lenguas.

\section{¿Cuál es el objetivo de este estudio?}

En esta ocasión, nos gustaría contar con tu ayuda para una investigación que lleva por título: "Hacia la creación de un Corpus del Profesorado de Español/LE - CoPELE". En ella, deseamos valorar la necesidad de la creación de un corpus colaborativo en el que se recojan las creencias, percepciones y actitudes del profesorado de ELE.

\section{¿Qué necesitamos?}

Si eres docente de español, te agradeceríamos que cumplimentaras en menos de diez minutos este breve cuestionario mediante el cual, de manera anónima y voluntaria, podremos conocer tu opinión acerca de la viabilidad y utilidad de la creación de un corpus de estas características.

Muchas gracias por el tiempo e interés mostrados.

\section{I) BIODATA}

Las siguientes preguntas nos permitirán saber más acerca de tu perfil como profesor de ELE.

\section{1. ¿Eres*}

profesora de español/lengua extranjera?

profesor de español/lengua extranjera?

\section{Lengua/s materna/s: *}

3. ¿Qué otras lenguas dominas? Indica la lengua y el nivel: Básico (B), Intermedio (I) o Avanzado (A). P. ej. inglés (A)

4. ¿Cuánto tiempo llevas impartiendo docencia de ELE? *

Menos de 1 año

Entre 1 y 5 años

Más de 5 años 


\section{5. ¿Has impartido docencia de otras lenguas? *} Sí

No

En caso afirmativo, ¿de cuáles?

6. ¿En qué lugares impartes/has impartido tus clases de español? * Puedes seleccionar más de una opción.

$\begin{array}{ll}\text { País/es hispanohablante/s } & \text { Asia } \\ \text { Europa } & \text { África } \\ \text { América } & \text { Oceanía }\end{array}$

7. ¿En qué niveles impartes clases de ELE? *

$\begin{array}{ll}\text { Enseñanza primaria } & \text { Academias y centros privados } \\ \text { Enseñanza secundaria } & \text { Clases particulares } \\ \text { Enseñanza universitaria } & \text { Otros }\end{array}$

En caso de otros, ¿cuáles?

8. ¿Has realizado alguna investigación relacionada con ELE? * Sí

No

En caso afirmativo, ¿puedes indicar sobre qué tema/s?

\section{II) UN CORPUS DEL PROFESORADO DE ESPAÑOL}

A continuación, te mostramos enunciados que debes valorar en una escala del 1 al 5 (1Muy en desacuerdo y 5-Totalmente de acuerdo). Los enunciados son relativos al uso de corpus y su necesidad en el aula de ELE.

A) El uso de corpus en el ámbito de enseñanza de ELE

1. Conozco algunos corpus del español. *

2. En el aula, empleo los corpus con fines pedagógicos. *

3. No poseo información sobre los corpus en la enseñanza de ELE*

4. Tengo claro cuál es el beneficio que puedo obtener de los corpus. *

5. En los cursos de formación del profesorado de ELE he recibido información sobre el uso de corpus en el aula de idiomas. *

6. Creo que es importante, como docente, llevar a cabo investigaciones en el aula de ELE.

7. Como parte de mi profesión, realizo una práctica reflexiva de enseñanza (a través de notas, de un diario, de un blog, etc.) *

8. Me interesan los aspectos de variables individuales en la enseñanza de lenguas. *

9. Si existiese un corpus sobre las creencias, percepciones y prácticas del profesorado de ELE, recurriría a él. *

10. Considero que la información que recogen los corpus es esencial para los profesores de ELE, independientemente de sus intereses en investigación. * 


\section{B) La elaboración de un corpus del profesorado de ELE}

A continuación, te solicitamos valores en una escala del 1 al 5 (1-Muy en desacuerdo y 5-Totalmente de acuerdo). Los enunciados son relativos a la viabilidad de crear un corpus sobre las creencias del profesorado de ELE.

1. La figura del profesorado de español goza de reconocimiento social. *

2. Es necesario investigar más en el perfil del profesorado para el reconocimiento de su estatus a nivel profesional. *

3. No creo que un corpus repercuta positivamente en mi ejercicio profesional. *

4. Me gustaría saber lo que otros profesores de ELE opinan sobre su ejercicio docente para ver si mis creencias, percepción y actitudes son o no individuales. *

5. Conocer más aspectos sobre las creencias de otro profesorado de ELE me ayudaría a reflexionar más sobre mis propias creencias en la enseñanza de ELE*

6. Saber más acerca de las creencias del profesorado permitiría conocer mejor sus necesidades formativas. *

7. Las redes sociales (grupos de Facebook, Twitter, blogs, foros, etc.) cubren gran parte de las necesidades que el profesorado posee acerca de las creencias y percepciones que estos poseen sobre la enseñanza de ELE. *

8. No veo la necesidad de recabar información acerca de las percepciones del profesorado. *

9. Cuando deseo averiguar aspectos sobre las creencias del profesorado de ELE, consulto/pregunto a través de las redes sociales. ${ }^{*}$

\section{C) Los beneficios de un corpus del profesorado de ELE}

$\mathrm{Si}$ consideras que un corpus que recoja las percepciones, actitudes y actuaciones del profesorado de ELE es útil para tu ejercicio profesional, ¿en qué medida crees que afectaría a las siguientes competencias del profesorado (Instituto Cervantes, 2012)? (1- Muy en desacuerdo, 5- Totalmente de acuerdo).

Creo que la existencia de un corpus del profesorado de español me ayudaría a...

1. Diagnosticar y atender las necesidades de los alumnos.

2. Promover el uso y la reflexión sobre la lengua. *

3. Planificar secuencias didácticas. *

4. Gestionar mi aula. *

5. Servirme de herramientas y procedimientos de evaluación. *

6. Garantizar buenas prácticas en la evaluación. *

7. Promover una retroalimentación constructiva. *

8. Implicar al alumno en la evaluación. *

9. Promover que el alumno gestione los recursos y medios disponibles para aprender. 
10. Integrar en la enseñanza herramientas para reflexionar sobre el proceso de aprendizaje. *

11. Promover que el alumno defina su propio proyecto de aprendizaje. *

12. Motivar al alumno para que se responsabilice de su propio aprendizaje. *

13. Desarrollar la propia competencia intercultural. *

14. Adaptarse a las culturas del entorno. *

15. Fomentar el diálogo intercultural. *

16. Promover que el alumno desarrolle su competencia intercultural. *

17. Analizar y reflexionar sobre la práctica docente. *

18. Definir un plan personal de formación continua. *

19. Implicarse en el desarrollo profesional del equipo docente. *

20. Participar activamente en el desarrollo de mi profesión. *

21. Gestionar mis propias emociones. *

22. Motivarme en el trabajo. *

23. Desarrollar las relaciones interpersonales. *

24. Implicarme en el desarrollo de la inteligencia emocional del alumno. *

25. Trabajar en equipo en el centro. *

26. Implicarme en los proyectos de mejora del centro. *

27. Promover y difundir buenas prácticas en la institución. *

28. Conocer la institución e integrarme en ella. *

29. Implicarme en el desarrollo de la propia competencia digital. *

30. Desenvolverme en entornos digitales y con aplicaciones informáticas disponibles. *

31. Aprovechar el potencial didáctico de las TIC. *

32. Promover que el alumno se sirva de las TIC para su aprendizaje. *

\section{¡Muchas gracias por completar el formulario!}

Por favor, si tienes alguna duda, sugerencia o consulta que realizarnos, puedes dejarnos un mensaje a continuación o escribirnos un correo electrónico a alberto.rodriguez@ua.es. 